\title{
Existence and multiplicity of periodic solutions for a generalized hematopoiesis model
}

\author{
Pablo Amster and Rocío Balderrama* \\ Departamento de Matemática, Facultad de Ciencias Exactas y Naturales \\ Universidad de Buenos Aires 8 IMAS-CONICET - Argentina.
}

\begin{abstract}
A generalization of the nonautonomous Mackey-Glass equation for the regulation of the hematopoiesis with several non-constant delays is studied. Using topological degree methods we prove, under appropriate conditions, the existence of multiple positive periodic solutions. Moreover, we show that the conditions are necessary, in the sense that if some sort of complementary conditions are assumed then the trivial equilibrium is a global attractor for the positive solutions and hence periodic solutions do not exist.
\end{abstract}

Keywords - Nonlinear nonautonomous delay differential equations; Positive periodic solutions; Multiplicity; Global attractor; Degree theory; Hematopoiesis.

\section{Introduction}

The following nonlinear autonomous delay differential equation was proposed by Mackey and Glass [11] to study the regulation of hematopoiesis:

$$
\frac{d P(t)}{d t}=\frac{\lambda \theta^{n} P(t-\tau)}{\theta^{n}+P^{n}(t-\tau)}-\gamma P(t) .
$$

Here $\lambda, \theta, n, \gamma, \tau$ are positive constants, $P(t)$ is the concentration of cells in the circulating blood and the flux function $f(v)=\frac{\lambda \theta^{n} v}{\theta^{n}+v^{n}}$ of cells into the blood stream depends on the cell concentration at an earlier time. The delay $\tau$ describes the

*This work was fully supported by project UBACyT 20020120100029BA. 
time between the start of cellular production in the bone marrow and the release of mature cells into the blood. It is assumed that the cells are lost at a rate proportional to their concentration, namely $\gamma P(t)$, where $\gamma$ is the decay rate. This equation constitutes a model of a 'dynamic disease'. This type of equation for population dynamics has attracted the interest of many researchers. Different aspects and properties of (1) have been studied by various authors, see for example $[5,6,10]$.

Most often, the environment varies with time; thus, it is intuitive to assume that this fact influences many biological dynamical systems and suggests the need of considering time-dependent parameters. Moreover, as remarked in $[4,8,16]$, more realistic models are those in which periodicity of the environment and time delay play a role (for more details, see e.g. [12]). In view of this, the following model was proposed in [16]:

$$
x^{\prime}(t)=\frac{q(t) x(t)}{r+x^{n}(t-m T)}-p(t) x(t)
$$

where $m$ and $n$ are positive integers, $p$ and $q$ are positive $T$-periodic functions and the delay $\tau:=m T$ is a multiple of the period determined by the environment.

In order to establish a more realistic model, it is convenient to introduce a more general delay that extends the two above-referred cases. Instead of assuming that the delay is constant or a multiple of the period of the environment, more general models are obtained by assuming that the time delay $\tau$ is an arbitrary continuous nonnegative $T$-periodic function depending on $t$. The more general equation

$$
x^{\prime}(t)=\frac{a(t) x(t-\tau(t))}{1+x^{n}(t-\tau(t))}-b(t) x(t)
$$

where $a, b$ and $\tau$ are continuous positive $T$-periodic functions was studied for example in [17-21]. Different aspects of equation (3) have been considered; in particular, existence of positive $T$-periodic solutions was proven, in most cases, using appropriate fixed point theorems. In [20], coincidence degree theory was employed to prove the existence of a positive $T$-periodic solution under a condition that can be regarded as a particular application of Theorem 3.2, case (2) below. Moreover, when $a(t)=\gamma b(t)$ for some $\gamma>0$ and when $\tau, a$ and $b$ are constant, the conditions $\gamma>1$ and $a>b$ respectively are both necessary and sufficient for the existence of positive $T$-periodic solutions.

The following more general model was studied in [2] and [9]:

$$
x^{\prime}(t)=\sum_{k=1}^{M} \frac{r_{k}(t) x^{\delta}\left(t-g_{k}(t)\right)}{1+x^{\gamma}\left(t-g_{k}(t)\right)}-b(t) x(t) .
$$


Here, $\gamma$ is a positive constant and $r_{k}, b$ are positive $T$-periodic continuous functions. For $\delta=1$, existence and uniqueness of positive $T$-periodic solutions was studied in [2] for the particular case of constant proportional delays $g_{k} \equiv l_{k} T$; moreover, for general continuous, positive $T$-periodic $g_{k}$, attractiveness of some specific positive periodic solutions was studied. For the case $\delta=0$ and $g_{k}$ continuous positive and $T$-periodic, existence and uniqueness of positive $T$-periodic solutions of (4) was proven in [9] by fixed point methods, provided that one of the following conditions is satisfied:

$$
\text { (1) } \gamma \leq 1 \text { or }(2) \gamma>1 \text { and }\left(\frac{e^{\int_{0}^{T} b(u) d u}}{e^{\int_{0}^{T} b(u) d u}-1} \int_{0}^{T} \sum_{k=1}^{M} r_{k}(t) d t\right)^{\gamma} \leq \frac{1}{\gamma-1} \text {. }
$$

Motivated by the previous discussion, we shall consider the following more general nonlinear nonautonomous model with several delays

$$
x^{\prime}(t)=\sum_{k=1}^{M} \lambda_{k} r_{k}(t) \frac{x^{m_{k}}\left(t-\tau_{k}(t)\right)}{1+x^{n_{k}}\left(t-\mu_{k}(t)\right)}-b(t) x(t)
$$

where $r_{k}(t), b(t), \tau_{k}(t)$ and $\mu_{k}(t)$ are positive and $T$-periodic functions and $\lambda_{k}, m_{k}, n_{k}$ are positive constants.

Existence of solutions of (5) under appropriate conditions follows from several abstract results, although multiplicity results are more scarce. For example, in [7] and [21] a Krasnoselskii type fixed point theorem in cones was employed in order to obtain conditions for the existence of at least two $T$-periodic solutions of the general equation

$$
x^{\prime}(t)=-a(t) x(t)+f\left(t, x\left(t-\tau_{1}(t)\right), \ldots, x\left(t-\tau_{n}(t)\right)\right) .
$$

It is observed, however, that these results can be applied only to some specific particular sub-cases of (5). In such cases, the conclusions are comparable to our results below. Moreover, the existence of three nonnegative periodic solutions of (6) was studied by using Leggett-Williams fixed point theorem in [3,13-15]. However, the conditions obtained in [13], as pointed out by the authors, are very difficult to apply to (5) with $M=1, m=1, \tau=\mu$. Thus, they established a complementary result with more straightforward conditions that can be applied to this model. Unfortunately, in [14], the authors observed that this latter result was incorrect. In section [14, Applications], the hematopoiesis model (5) for $M=1, \tau=\mu$ was studied. The conditions obtained by the authors are similar to the ones proposed in Theorem 4.2 (1) below, although only two of the three $T$-periodic solutions are positive and the third one is positive if $f(t, 0)$ is not identically zero. This assumption is very restrictive and clearly not fulfilled in (5). We may also mention the 
work [22], in which the existence of at least $2 n$ solutions of (6) is proven, although the conditions are not applicable to our model. Moreover, all the mentioned works do not contemplate the superlinear case of (5) (that is, $m_{k}>n_{k}+1$ for some $k$ ). From the biological point of view, this makes sense since the nonlinearity is a measure of the cellular production in the bone marrow, and therefore it should be bounded; however, the superlinear case is also of mathematical interest in order to obtain a complete picture of the different cases in (5).

Our goal in this paper is to establish sufficient criteria to guarantee, on the one hand, the existence of positive $T$-periodic solutions of (5) and, on the other hand, multiplicity of such solutions. Using degree theory, we shall obtain a set of natural and easy-to-verify conditions for the existence of one or more solutions. Moreover, in some cases we shall also find necessary conditions for the existence of positive periodic solutions. More precisely, we shall establish conditions that are incompatible with those obtained for the existence results and imply that all positive solutions tend to 0 as $t \rightarrow+\infty$.

The paper is organized as follows. In the next section, we introduce some preliminary results and notation that shall be used throughout the paper. In Section 3 we apply Theorem 2.1 in order to prove the existence of positive $T$ periodic solutions for the different cases. In Section 4, we give sufficient conditions for the existence of 2, 3 or 4 positive $T$-periodic solutions. In Section 5 we give an example with at least 6 positive $T$-periodic solutions. Finally, in Section 6 we establish necessary conditions for the existence of positive periodic solutions. More precisely, we prove that, under an appropriate assumption, all positive solutions of (5) tend to 0 as $t \rightarrow \infty$ and, consequently, they cannot be periodic.

\section{Preliminaries.}

The following notation shall be used throughout the paper. Let

$$
C_{T}:=\{u(t) \in C(\mathbb{R}, \mathbb{R}): u(t+T)=u(t) \text { for all } t\}
$$

denote the space of continuous $T$-periodic functions and define, for $r<s$,

$$
X_{r}^{s}:=\left\{u(t) \in C_{T}: r<u(t)<s \text { for all } t\right\} .
$$

The closure of $X_{r}^{s}$ shall be denoted by $\operatorname{cl}\left(X_{r}^{s}\right)$. The average, the maximum value and the minimum value of an arbitrary function $\varphi \in C_{T}$ shall be denoted respectively by $\bar{\varphi}, \varphi_{\max }$ and $\varphi_{\min }$, namely

$$
\bar{\varphi}:=\frac{1}{T} \int_{0}^{T} \varphi(t) d t, \quad \varphi_{\max }=\max _{[0, T]} \varphi(t), \quad \varphi_{\min }=\min _{[0, T]} \varphi(t) .
$$


In order to simplify some computations, we set $y(t):=\ln (x(t))$ and transform (5) into the equivalent equation

$$
y^{\prime}(t)=\sum_{k=1}^{M} \lambda_{k} r_{k}(t) \frac{e^{m_{k} y\left(t-\tau_{k}(t)\right)-y(t)}}{1+e^{n_{k} y\left(t-\mu_{k}(t)\right)}}-b(t) .
$$

Finally we define, for convenience, the function $\phi: \mathbb{R} \rightarrow \mathbb{R}$ by

$$
\phi(\gamma):=\sum_{k=1}^{M} \lambda_{k} \bar{r}_{k} \frac{e^{\left(m_{k}-1\right) \gamma}}{1+e^{n_{k} \gamma}}-\bar{b} .
$$

The proof of our results shall be based on the continuation method. Specifically, we shall apply the following existence theorem, which can be directly deduced from Theorem 2.1 in [1].

Theorem 2.1 Assume there exist constants $\gamma_{1}<\gamma_{2}$ such that

1. If $y \in \operatorname{cl}\left(X_{\gamma_{1}}^{\gamma_{2}}\right)$ satisfies

$$
y^{\prime}(t)=\sigma\left(\sum_{k=1}^{M} \lambda_{k} r_{k}(t) \frac{e^{m_{k} y\left(t-\tau_{k}(t)\right)-y(t)}}{1+e^{n_{k} y\left(t-\mu_{k}(t)\right)}}-b(t)\right)
$$

for some $\sigma \in(0,1]$, then $y \in X_{\gamma_{1}}^{\gamma_{2}}$.

2. $\phi\left(\gamma_{1}\right) \phi\left(\gamma_{2}\right)<0$.

Then (7) has at least one solution in $X_{\gamma_{1}}^{\gamma_{2}}$.

Roughly speaking, if $\phi$ has different signs at both ends of some interval $\left[\gamma_{1}, \gamma_{2}\right] \subset$ $\mathbb{R}$ then the continuation theorem guarantees the existence of a $T$-periodic solution $y$ of $(7)$ such that $y(t) \in\left(\gamma_{1}, \gamma_{2}\right)$ for all $t$. However, the first condition of Theorem 2.1 requires, in some sense, that the sign of $\phi$ does not change too fast.

The main part of our analysis shall be based on a study of the behaviour of $\phi$. For a proof of the existence of at least one solution it suffices, in most cases, to consider its behavior at $\pm \infty$; for the multiplicity results, a more careful study is needed, in order to find intervals of positivity and negativity of $\phi$ that are sufficiently large, so the conditions of the continuation theorem can be fulfilled. With this end in mind, we shall consider the sets

$$
\begin{gathered}
M_{1}:=\left\{k: 0<m_{k}<1\right\}, M_{2}:=\left\{k: m_{k}=1\right\}, M_{3}:=\left\{k: 1<m_{k}<n_{k}+1\right\} \\
M_{4}:=\left\{k: m_{k}=n_{k}+1\right\}, M_{5}:=\left\{k: m_{k}>n_{k}+1\right\}
\end{gathered}
$$


and the mappings

$$
\phi_{i}(\gamma):=\sum_{k \in M_{i}} \lambda_{k} \bar{r}_{k} \frac{e^{\left(m_{k}-1\right) \gamma}}{1+e^{n_{k} \gamma}},
$$

so we may write $\phi(\gamma)=\sum_{i=1}^{5} \phi_{i}(\gamma)-\bar{b}$. For notation convenience, we also define $B:=T \bar{b}=\int_{0}^{T} b(t) d t$.

This setting proves to be useful, since the limits $\lim _{\gamma \rightarrow \pm \infty} \phi_{i}(\gamma)$ are easy to compute and, moreover, $\phi_{i}(\gamma)$ is strictly monotone for $i \neq 3$ and a sum of onehump functions for $i=3$. Thus, the behavior of $\phi$ can be understood by studying the interaction of these different terms.

\section{Existence of positive $T$-periodic solutions.}

In order to present our existence results in a more comprehensive way, we shall consider three different cases: the superlinear case $\left(m_{k}>n_{k}+1\right.$ for some $\left.k\right)$, the the sublinear case $\left(m_{k}<n_{k}+1\right.$ for all $\left.k\right)$ and the asymptotically linear case $\left(m_{k} \leq n_{k}+1\right.$ for all $k$ and $m_{j}=n_{j}+1$ for some $j$ ). We give a detailed proof only of the first result, since the other two follow similarly.

Theorem 3.1 Assume $m_{j}>n_{j}+1$ for some $j$. Furthermore, assume that one of the following conditions is fulfilled:

1. $m_{k}>1$ for all $k$.

2. $m_{k} \geq 1$ for all $k, m_{i}=1$ for some $i$ and $\sum_{k \in M_{2}} \lambda_{k} r_{k}(t) e^{B}<b(t)$ for all $t$.

3. $m_{i}<1$ for some $i$ and $\sum_{k=1}^{M} \lambda_{k} r_{k}(t) \frac{e^{\left(m_{k}-1\right) \gamma_{1} e^{m_{k} B}}}{1+e^{n_{k} \gamma_{1}}}<b(t)$ for all $t$ and some constant $\gamma_{1}$.

Then (5) admits at least one positive T-periodic solution.

Theorem 3.2 Assume $m_{k}<n_{k}+1$ for all $k$. Furthermore, assume that one of the following conditions is fulfilled:

1. $m_{i}<1$ for some $i$.

2. $m_{k} \geq 1$ for all $k, m_{i}=1$ for some $i$ and $\sum_{k \in M_{2}} \lambda_{k} r_{k}(t)>b(t)$ for all $t$.

3. $m_{k}>1$ for all $k$ and

$$
\sum_{k=1}^{M} \lambda_{k} r_{k}(t) \frac{e^{\left(m_{k}-1\right) \gamma_{1}}}{1+e^{n_{k}\left(\gamma_{1}+B\right)}}>b(t)
$$

for all $t$ and some arbitrary constant $\gamma_{1}$. 
Then (5) admits at least one positive T-periodic solution.

Theorem 3.3 Assume $m_{k} \leq n_{k}+1$ for all $k$ and $m_{j}=n_{j}+1$ for some $j$. Furthermore, assume that one of the following conditions is fulfilled:

1. $m_{k}>1$ for all $k$ and $\sum_{k \in M_{4}} \lambda_{k} r_{k}(t) e^{-B m_{k}}>b(t)$ for all $t$.

2. $m_{k} \geq 1$ for all $k, m_{i}=1$ for some $i, \sum_{k \in M_{4}} \lambda_{k} r_{k}(t) e^{-B m_{k}}>b(t)$ and $\sum_{k \in M_{2}} \lambda_{k} r_{k}(t) e^{B}<b(t)$ for all $t$.

3. $0<m_{i}<1$ for some $i$ and $\sum_{m_{k} \in M_{4}} \lambda_{k} r_{k}(t) e^{B n_{k}}<b(t)$ for all $t$.

Then (5) admits at least one positive T-periodic solution.

Proof of Theorem 3.1: Let $y$ be a $T$-periodic solution of (9) with $0<\sigma \leq 1$, then

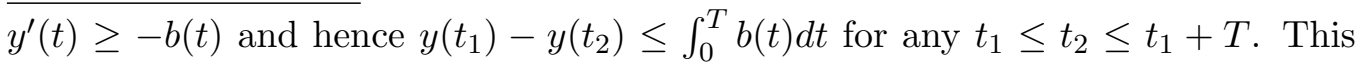
implies, since $y(t)$ is $T$-periodic, that $y_{\max }-y_{\min } \leq \int_{0}^{T} b(t) d t=B$. Moreover, since $m_{k}>n_{k}+1$ for some $k$ it follows that $\phi(\gamma)>0$ when $\gamma$ is large enough. Assume that $y_{\max }$ is achieved at some value $t^{*}$, that is $y\left(t^{*}\right)=y_{\max }$, then $y^{\prime}\left(t^{*}\right)=0$. Hence from (9) we deduce, since $\sigma>0$,

$$
\begin{aligned}
& b\left(t^{*}\right) e^{y_{\max }}=\sum_{k=1}^{M} \lambda_{k} r_{k}\left(t^{*}\right) \frac{e^{m_{k} y\left(t^{*}-\tau_{k}\left(t^{*}\right)\right)}}{1+e^{n_{k} y\left(t^{*}-\mu_{k}\left(t^{*}\right)\right)}} \\
& \geq \sum_{k=1}^{M} \lambda_{k} r_{k}\left(t^{*}\right) \frac{e^{m_{k}\left(y_{\max }-B\right)}}{1+e^{n_{k} y_{\max }}}
\end{aligned}
$$

and consequently

$$
b\left(t^{*}\right) \geq \sum_{k=1}^{M} \lambda_{k} r_{k}\left(t^{*}\right) \frac{e^{\left(m_{k}-1\right) y_{\max }} e^{-B m_{k}}}{1+e^{n_{k} y_{\max }}} .
$$

Again, since $m_{k}>n_{k}+1$ for some $k$ we deduce that $y_{\max }$ cannot be too large. Thus, we may fix $\gamma_{2} \gg 0$ such that $y_{\max }<\gamma_{2}$ for every $y \in C_{T}$ satisfying (9) and $\phi\left(\gamma_{2}\right)>0$. In a similar fashion, we look for $\gamma_{1}<\gamma_{2}$ such that $\phi\left(\gamma_{1}\right)<0$ and $y_{\text {min }} \neq \gamma_{1}$.

Case 1: $m_{k}>1$ for all $k$. Here

$$
\phi(\gamma) \rightarrow-\bar{b} \quad \text { as } \gamma \rightarrow-\infty
$$


Let $y \in C_{T}$ be a solution of (9) and fix $t_{*}$ such that $y\left(t_{*}\right)=y_{\min }$, then

$$
b\left(t_{*}\right) \leq \sum_{k=1}^{M} \lambda_{k} r_{k}\left(t_{*}\right) \frac{e^{\left(m_{k}-1\right) y_{\min }} e^{B m_{k}}}{1+e^{n_{k} y_{\min }}}
$$

Suppose that $y_{\min }=\gamma_{1}$, then

$$
b\left(t_{*}\right) \leq \sum_{k=1}^{M} \lambda_{k} r_{k}(t) \frac{e^{\left(m_{k}-1\right) \gamma_{1}+B m_{k}}}{1+e^{n_{k} \gamma_{1}}} .
$$

The right-hand side of the latter inequality tends to zero as $\gamma_{1} \rightarrow-\infty$. We deduce that $y_{\min }$ cannot take arbitrarily large negative values; hence, it suffices to take $\gamma_{1} \ll 0$.

Case 2. $m_{k} \geq 1$ for all $k$ and $m_{j}=1$ for some $j$. In this case,

$$
\phi(\gamma) \rightarrow \sum_{k \in M_{2}} \lambda_{k} \overline{r_{k}}-\bar{b}<\sum_{k \in M_{2}} \lambda_{k} \overline{r_{k}} e^{B}-\bar{b}<0
$$

as $\gamma \rightarrow-\infty$. On the other hand, if $y \in C_{T}$ satisfies (9) then

$$
b\left(t_{*}\right) \geq \sum_{k \in M_{2}} \lambda_{k} r_{k}\left(t_{*}\right) \frac{e^{\left(m_{k}-1\right) y_{\text {min }}+B m_{k}}}{1+e^{n_{k}\left(y_{\min }+B\right)}}=\sum_{k \in M_{2}} \lambda_{k} r_{k}\left(t_{*}\right) \frac{e^{B}}{1+e^{n_{k}\left(y_{\min }+B\right)}}
$$

and, again, we deduce that $y_{\min }$ cannot take too large negative values. Thus, it suffices to take $\gamma_{1} \ll 0$.

Case 3. $m_{k}<1$ for some $k$. From the hypothesis,

$$
\phi\left(\gamma_{1}\right)=\sum_{k=1}^{M} \lambda_{k} \bar{r}_{k} \frac{e^{\left(m_{k}-1\right) \gamma_{1}}}{1+e^{n_{k} \gamma_{1}}}-\bar{b} \leq \sum_{k=1}^{M} \lambda_{k} \bar{r}_{k} \frac{e^{\left(m_{k}-1\right) \gamma_{1}} e^{B m_{k}}}{1+e^{n_{k} \gamma_{1}}}-\bar{b}<0 .
$$

Moreover, if $y_{\min }$ is achieved at some value $t_{*}$, then

$$
b\left(t_{*}\right) \leq \sum_{k=1}^{M} \lambda_{k} r_{k}\left(t_{*}\right) \frac{e^{\left(m_{k}-1\right) y_{\min }} e^{B m_{k}}}{1+e^{n_{k} y_{\min }}} .
$$

We conclude that $y_{\min } \neq \gamma_{1}$.

Remark 3.1 It is easy to verify that the second condition in Theorem 3.3 can be replaced by

2'. $m_{k} \geq 1$ for all $k, m_{i}=1$ for some $i, \sum_{k \in M_{4}} \lambda_{k} r_{k}(t) e^{B n_{k}}<b(t)$ and $\sum_{k \in M_{2}} \lambda_{k} r_{k}(t)>$ $b(t)$ for all $t$. 


\section{Multiplicity}

In this section, we shall employ Theorem 2.1 in order to prove the existence of multiple solutions. It is worth noticing that, when $\phi$ is monotone, it changes sign at most once and the method cannot be applied. On the other hand, when $\phi$ is non-monotone, it is not enough to obtain intervals of positivity and negativity: as mentioned, it is required that $\phi$ does not change sign too fast. For a more detailed analysis, the following functions shall be helpful:

$$
\begin{aligned}
\alpha(\gamma, t) & :=\sum_{k=1}^{M} \lambda_{k} r_{k}(t) \frac{e^{\left(m_{k}-1\right) \gamma} e^{-B m_{k}}}{1+e^{n_{k}(\gamma+B)}}-b(t) \\
\beta(\gamma, t) & :=\sum_{k=1}^{M} \lambda_{k} r_{k}(t) \frac{e^{\left(m_{k}-1\right) \gamma} e^{B m_{k}}}{1+e^{n_{k}(\gamma-B)}}-b(t)
\end{aligned}
$$

As before, our results shall be presented in three different theorems, for the superlinear, sublinear and asymptotically linear cases.

Theorem 4.1 Assume that $m_{j}>n_{j}+1$ for some $j$.

1. Let $m_{k}>1$ for all $k$ and $1<m_{i}<n_{i}+1$ for some $i$. Assume there exist constants $\gamma_{1}<\gamma_{2}$ such that

$$
\alpha\left(\gamma_{1}, t\right)>0>\beta\left(\gamma_{2}, t\right) \text { for all } t .
$$

Then (5) admits at least 3 positive T-periodic solutions.

2. Let $m_{k} \geq 1$ for all $k, m_{i}=1$ for some $i, m_{k} \notin\left(1, n_{k}+1\right)$ for all $k$. Assume that

$$
\sum_{k \in M_{2}} \lambda_{k} r_{k}(t)>b(t) \text { for all } t
$$

and there exists $\gamma_{1}$ such that

$$
\beta\left(\gamma_{1}, t\right)<0 \text { for all } t .
$$

Then (5) admits at least 2 positive T-periodic solutions.

3. Let $m_{k} \geq 1$ for all $k, m_{i}=1$ for some $i$ and $1<m_{s}<n_{s}+1$ for some $s$. Assume that

$$
\sum_{k \in M_{2}} \lambda_{k} r_{k}(t) e^{B}<b(t) \text { for all } t
$$


and there exist constants $\gamma_{1}<\gamma_{2}$ such that

$$
\alpha\left(\gamma_{1}, t\right)>0>\beta\left(\gamma_{2}, t\right) \text { for all } t .
$$

Then (5) admits at least 3 positive T-periodic solutions.

4. Let $m_{i}<1$ for some $i, m_{k} \notin\left(1, n_{k}+1\right)$ for all $k$. Assume

$$
\beta\left(\gamma_{1}, t\right)<0 \text { for all } t \text { and some constant } \gamma_{1} \text {. }
$$

Then (5) admits at least 2 positive T-periodic solutions.

5. Let $m_{i}<1$ for some $i, 1<m_{s}<n_{s}+1$ for some $s$. Assume there exist some constants $\gamma_{1}<\gamma_{2}<\gamma_{3}$ such that

$$
\alpha\left(\gamma_{2}, t\right)>0>\beta\left(\gamma_{i}, t\right) \text { for all } t,
$$

for $i=1,3$. Then (5) admits at least 4 positive T-periodic solutions.

Theorem 4.2 Assume that $m_{k}<n_{k}+1$ for all $k$.

1. Let $m_{k}>1$ for all $k$ and assume there exists a constant $\gamma_{1}$ such that

$$
\alpha\left(\gamma_{1}, t\right)>0 \text { for all } t .
$$

Then (5) admits at least 2 positive T-periodic solutions.

2. Let $m_{k} \geq 1$ for all $k, m_{i}=1, m_{j}>1$ for some $i, j$. Assume that

$$
\sum_{k \in M_{2}} \lambda_{k} r_{k}(t) e^{B}<b(t) \text { for all } t
$$

and there exists a constant $\gamma_{1}$ such that

$$
\alpha\left(\gamma_{1}, t\right)>0 \text { for all } t .
$$

Then (5) admits at least 2 positive T-periodic solutions.

3. Let $0<m_{i}<1, m_{j}>1$ for some $i, j$. Assume there exist some constants $\gamma_{1}<\gamma_{2}$ such that

$$
\alpha\left(\gamma_{2}, t\right)>0>\beta\left(\gamma_{1}, t\right) \text { for all } t .
$$

Then (5) admits at least 3 solutions. 
Theorem 4.3 Assume that $m_{k} \leq n_{k}+1$ for all $k$ and $m_{j}=n_{j}+1$ for some $j$.

1. Let $m_{k}>1$ for all $k$ and $1<m_{i}<n_{i}+1$ for some $i$. Assume that

$$
\sum_{k \in M_{4}} \lambda_{k} r_{k}(t) e^{B n_{k}}<b(t) \text { for all } t
$$

and there exists a constant $\gamma_{1}$ such that

$$
\alpha\left(\gamma_{1}, t\right)>0 \text { for all } t .
$$

Then (5) admits at least 2 positive T-periodic solutions.

2. Let $0<m_{i}<1$ for some $i, m_{k} \notin\left(1, n_{k}+1\right)$ for all $k$. Assume that

$$
\sum_{k \in M_{4}} \lambda_{k} r_{k}(t) e^{-B m_{k}}>b(t) \text { for all } t
$$

and there exists $\gamma_{1}$ such that

$$
\beta\left(\gamma_{1}, t\right)<0 \text { for all } t .
$$

Then (5) admits at least 2 positive T-periodic solutions.

3. Let $0<m_{i}<1$ and $1<m_{s}<n_{s}+1$ for some $i, s$. Assume that

$$
\sum_{k \in M_{4}} \lambda_{k} r_{k}(t) e^{B n_{k}}<b(t)
$$

and there exist constants $\gamma_{1}<\gamma_{2}$ such that

$$
\alpha\left(\gamma_{2}, t\right)>0>\beta\left(\gamma_{1}, t\right) \text { for all } t .
$$

Then (5) has at least 3 positive T-periodic solutions.

As before, we shall only prove the first case of Theorem 4.1, since all the remaining cases follow in an analogous way.

Proof of Theorem 4.1, case 1: We shall apply Theorem 2.1 on open bounded sets

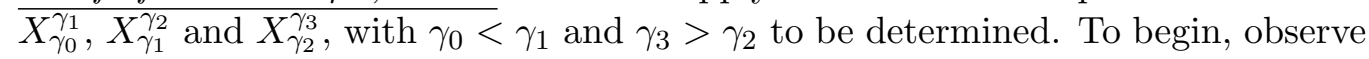
that

$$
\phi(\gamma) \rightarrow-\bar{b} \quad \text { as } \gamma \rightarrow-\infty
$$

and 


$$
\phi(\gamma) \rightarrow+\infty \quad \text { as } \gamma \rightarrow+\infty .
$$

In the same way of Theorem 3.1 it is proven that, if $\gamma_{0} \ll 0$ then there exists $y \in X_{\gamma_{0}}^{\gamma_{1}}$ solution of (7).

On the other hand, for all $t$ it is seen that

$$
\phi\left(\gamma_{1}\right)>\alpha\left(\gamma_{1}, t\right)>0
$$

Moreover, if $y \in \operatorname{cl}\left(X_{\gamma_{1}}^{\gamma_{2}}\right)$ is a solution of (9) with $0<\sigma \leq 1$ and $y_{\min }=y\left(t_{*}\right)$, then

$$
\begin{gathered}
b\left(t_{*}\right) e^{y_{\min }}=\sum_{k=1}^{M} \lambda_{k} r_{k}\left(t_{*}\right) \frac{e^{m_{k} y\left(t_{*}-\tau_{k}\left(t_{*}\right)\right)}}{1+e^{n_{k} y\left(t_{*}-\mu_{k}\left(t_{*}\right)\right)}} \\
>\sum_{k=1}^{M} \lambda_{k} r_{k}\left(t_{*}\right) \frac{e^{m_{k} y_{\min }}}{1+e^{n_{k}\left(y_{\min }+B\right)}}>\sum_{k=1}^{M} \lambda_{k} r_{k}\left(t_{*}\right) \frac{e^{m_{k} y_{\text {min }}} e^{-B m_{k}}}{1+e^{n_{k}\left(y_{\min }+B\right)}} .
\end{gathered}
$$

It follows that $y_{\min } \neq \gamma_{1}$.

Furthermore,

$$
\phi\left(\gamma_{2}\right)<\beta\left(\gamma_{2}, t\right)<0
$$

for all $t$ and we deduce as before that $y_{\max } \neq \gamma_{2}$.

Finally, the existence of $\gamma_{3} \gg 0$ such that the problem has a solution $y \in X_{\gamma_{2}}^{\gamma_{3}}$ follows as in Theorem 3.1.

The following lemma shows, in the context of Theorem 4.1 (case 1), that if $r_{k}, m_{k}$ and $n_{k}$ are given, then it is possible to find parameters $\lambda_{k}$ such that assumptions are fulfilled. Analogous arguments are valid for the remaining cases.

Lemma 4.1 Let $r_{k}, b: \mathbb{R} \rightarrow \mathbb{R}_{>0}$ be continuous and $T$-periodic functions and $m_{k}, n_{k} \in \mathbb{R}_{>0}$ such that $m_{k}>1$ for all $k, 1<m_{j}<n_{j}+1$ for some $j, m_{i}>n_{i}+1$ for some $i$. Then there exist $\lambda_{k}$ and $\gamma_{1}<\gamma_{2}$ such that

$$
\alpha\left(\gamma_{1}, t\right)>0>\beta\left(\gamma_{2}, t\right) \text { for all } t .
$$

Proof: Using the sets $M_{i}$ as before, we may write $\alpha$ and $\beta$ as

$$
\alpha(\gamma, t)=\sum_{i=1}^{5} \alpha_{i}(\gamma, t)-b(t), \quad \beta(\gamma, t)=\sum_{i=1}^{5} \beta_{i}(\gamma, t)-b(t)
$$

where

$$
\alpha_{i}(\gamma, t):=\sum_{k \in M_{i}} \lambda_{k} r_{k}(t) \frac{e^{\left(m_{k}-1\right) \gamma} e^{-B m_{k}}}{1+e^{n_{k}(\gamma+B)}}, \quad \beta_{i}(\gamma, t):=\sum_{k \in M_{i}} \lambda_{k} r_{k}(t) \frac{e^{\left(m_{k}-1\right) \gamma} e^{B m_{k}}}{1+e^{n_{k}(\gamma-B)}}
$$


Observe that, for each $t \in[0, T]$ and $i=1, \ldots, 5$, the functions $\alpha_{i}(\cdot, t)$ and $\beta_{i}(\cdot, t)$ have the same qualitative behavior as the mappings $\phi_{i}$ given by (10).

We begin by setting the parameters $\lambda_{k} \in M_{3}$. For arbitrary $\gamma_{1}$, take $\lambda_{k} \in M_{3}$ large enough such that

$$
\alpha\left(\gamma_{1}, t\right) \geq \sum_{k \in M_{3}} \lambda_{k} r_{k}(t) \frac{e^{\left(m_{k}-1\right) \gamma_{1}} e^{-B m_{k}}}{1+e^{n_{k}\left(\gamma_{1}+B\right)}}-b(t)>0 .
$$

For $\epsilon \in\left(0, b_{\text {min }}\right)$, there exists $R>\gamma_{1}$ such that

$$
\beta_{3}(\gamma, t)=\sum_{k \in M_{3}} \lambda_{k} r_{k}(t) \frac{e^{\left(m_{k}-1\right) \gamma} e^{B m_{k}}}{1+e^{n_{k}(\gamma-B)}}<\sum_{k \in M_{3}} \lambda_{k} r_{k}^{\max } \frac{e^{\left(m_{k}-1\right) \gamma} e^{B m_{k}}}{1+e^{n_{k}(\gamma-B)}}<\epsilon
$$

for $\gamma>R$ and all $t$. Thus, we may fix $\gamma_{2}>R$ and proceed with the remaining parameters.

Next, for $k \in M_{4} \cup M_{5}$ we set $\lambda_{k}$ small enough so that

$$
\sum_{k \in M_{4} \cup M_{5}} \lambda_{k}\left(r_{k}\right)_{\max } \frac{e^{\left(m_{k}-1\right) \gamma_{2}} e^{B m_{k}}}{1+e^{n_{k}\left(\gamma_{2}-B\right)}}<b_{\min }-2 \epsilon
$$

and hence

$$
\left(\beta_{4}+\beta_{5}\right)\left(\gamma_{2}, t\right)=\sum_{k \in M_{4} \cup M_{5}} \lambda_{k} r_{k}(t) \frac{e^{\left(m_{k}-1\right) \gamma_{2}} e^{B m_{k}}}{1+e^{n_{k}\left(\gamma_{2}-B\right)}}<b_{\text {min }}-2 \epsilon<b(t)-2 \epsilon .
$$

Thus the conclusion follows since

$$
\beta\left(\gamma_{2}, t\right)=\left(\beta_{3}+\beta_{4}+\beta_{5}\right)\left(\gamma_{2}, t\right)-b(t)<\epsilon-2 \epsilon<0
$$

and

$$
\begin{gathered}
\alpha\left(\gamma_{1}, t\right)=\left(\alpha_{3}+\alpha_{4}+\alpha_{5}\right)\left(\gamma_{1}, t\right)-b(t) \\
>\alpha_{3}\left(\gamma_{1}, t\right)-b(t)>0
\end{gathered}
$$

\section{Example}

As shown in Theorem 4.1, case 5, equation (5) has at least 4 positive $T$-periodic solutions. The following example shows that, in fact, the problem may have more solutions. Let $k=4$ and $b(t)=1.1+0.02 \cos \left(\frac{2 \pi t}{T}\right), T=0.005, m_{1}=0.95, n_{1}=2$, 
$\lambda_{1} r_{1}(t)=0.04+0.002 \cos \left(\frac{2 \pi t}{T}\right), m_{2}=4.73, n_{2}=3.74, \lambda_{2} r_{2}(t)=1.3+0.002 \cos \left(\frac{2 \pi t}{T}\right)$, $m_{3}=1.0001, n_{3}=10.2, \lambda_{3} r_{3}(t)=0.9+0.002 \cos \left(\frac{2 \pi t}{T}\right), m_{4}=1.12, n_{4}=0.11$, $\lambda_{4} r_{4}(t)=0.06+0.002 \cos \left(\frac{2 \pi t}{T}\right)$.

Set $\gamma_{1}=-5, \gamma_{2}=-0.3 \gamma_{3}=0.2, \gamma_{4}=5, \gamma_{5}=34$. It is verified (see Figure 1) that

$$
\alpha\left(\gamma_{2}, t\right)>0.09, \alpha\left(\gamma_{4}, t\right)>0.1 \text { for all } t
$$

and

$$
\beta\left(\gamma_{1}, t\right)<-0.08, \beta\left(\gamma_{3}, t\right)<-0.01, \beta\left(\gamma_{5}, t\right)<-0.01 \text { for all } t .
$$

Moreover, since $0<m_{1}=0.95<1$ and $m_{4}=1.12>n_{4}+1=1.11$, it follows that

$$
\lim _{\gamma \rightarrow-\infty} \phi(\gamma)=\lim _{\gamma \rightarrow+\infty} \phi(\gamma)=+\infty
$$

Thus, as in the previous proofs, we may set $\gamma_{0} \ll 0$ and $\gamma_{6} \gg 0$ in such a way that the problem has a solution in $X_{\gamma_{k}}^{\gamma_{k+1}}$ for $k=0, \ldots, 5$. We conclude that (5) has at least six positive 0.005-periodic solutions for arbitrary nonnegative 0.005-periodic delays $\tau_{k}, \mu_{k}$.
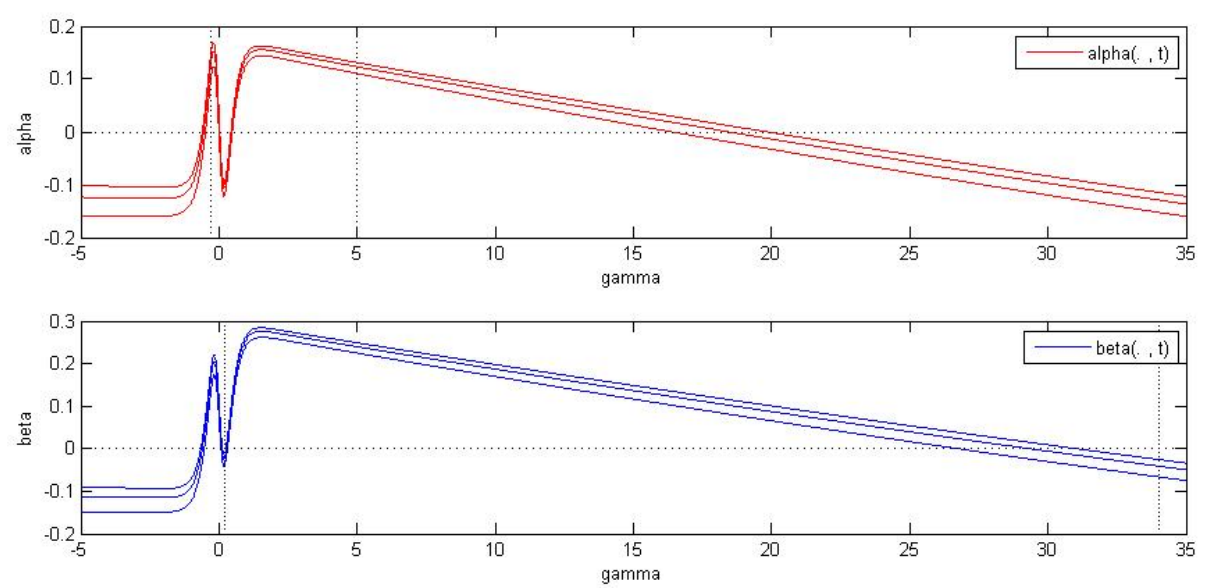

Figure 1: $\alpha(\cdot, t)$ and $\beta(\cdot, t)$ for each $t \in[0: 0.01: T]$. 


\section{$6 \quad$ Necessary conditions}

In this section we shall prove that, under certain assumptions, the trivial equilibrium is a global attractor for the positive solutions of (5). This proves, in particular, that positive $T$-periodic solutions cannot exist. As we shall see at the end of the section, both in the case that $m_{j}<1$ for some $j$ and the superlinear case, the autonomous problem admits positive equilibrium points and hence 0 cannot be a global attractor for the positive solutions. Hence, we shall consider only the case $1 \leq m_{k} \leq n_{k}+1$ for all $k$ and, thus, our non-existence result can be regarded, in some sense, as complementary to Theorem 3.2 (cases 2 and 3 ) and Theorem 3.3 (cases 1 and 2).

Throughout this section we shall assume that $\mu_{k}(t) \leq \tau_{k}(t)$. For convenience, we define $v:=\max _{1 \leq k \leq M, t \in \mathbb{R}}\left\{\tau_{k}(t)\right\}$

Remark 6.1 Let be $y(t)$ a solution of (7). Then

$$
y^{\prime}(t) \geq-b(t)
$$

and hence $y\left(t_{1}\right) \leq y\left(t_{2}\right)+\int_{t_{1}}^{t_{2}} b(t) d t$ for any $t_{1} \leq t_{2}$. In particular, this implies that

$$
y\left(t-\tau_{k}(t)\right), y\left(t-\mu_{k}(t)\right) \leq L+y(t),
$$

where $L=\max _{t \in[0, T]} \int_{t-v}^{t} b(s) d s$ and

$$
y\left(t-\tau_{k}(t)\right) \leq y\left(t-\mu_{k}(t)\right)+B_{k},
$$

where $B_{k}=\max _{t \in[0, T]} \int_{t-\tau_{k}(t)}^{t-\mu_{k}(t)} b(s) d s$.

Theorem 6.1 Let $1 \leq m_{k} \leq n_{k}+1$ for all $k$. Assume that

$$
\sum_{k=1}^{M} \lambda_{k} r_{k}(t) e^{m_{k} B_{k}} \leq b(t)
$$

Then every positive solution of (5) tends to 0 as $t \rightarrow+\infty$. In particular (5) has no positive T-periodic solutions.

Proof: We shall prove that every solution of (7) tends to $-\infty$ as $t \rightarrow+\infty$. To this end, let $y$ be a solution of (7) and suppose firstly that there exists a sequence $\left\{t_{j}\right\}_{j \in \mathbb{N}}$ such that $t_{j} \nearrow+\infty, y^{\prime}\left(t_{j}\right) \geq 0$, and $y(t)<y\left(t_{j}\right)$ for all $t_{1}-v \leq t<t_{j}$. It follows from (12)-(13) that

$$
0 \leq y^{\prime}\left(t_{j}\right) \leq \sum_{k=1}^{M} \lambda_{k} r_{k}\left(t_{j}\right) e^{m_{k} B_{k}} \frac{e^{m_{k} y\left(t_{j}-\mu_{k}\left(t_{j}\right)\right)-y\left(t_{j}\right)}}{1+e^{n_{k} y\left(t_{j}-\mu_{k}\left(t_{j}\right)\right)}}-b\left(t_{j}\right)
$$


and hence

$$
\begin{aligned}
0 & \leq \sum_{k=1}^{M} \lambda_{k} r_{k}\left(t_{j}\right) e^{m_{k} B_{k}} e^{y\left(t_{j}-\mu_{k}\left(t_{j}\right)\right)-y\left(t_{j}\right)}-b\left(t_{j}\right) \\
& \leq \sum_{k=1}^{M} \lambda_{k} r_{k}\left(t_{j}\right) e^{m_{k} B_{k}}-b\left(t_{j}\right)<0,
\end{aligned}
$$

a contradiction. We conclude that $y^{\prime}$ cannot be nonnegative on $\left(t_{0},+\infty\right)$ for any $t_{0}$ and $\lim \sup _{t \rightarrow+\infty} y(t):=\omega_{1}<+\infty$. Thus, we may consider the two possible cases:

Case I: There exists $t_{0}>0$ large enough such that $y^{\prime}(t) \leq 0$ for all $t \geq t_{0}$. It follows that $y(t) \rightarrow \alpha \in[-\infty,+\infty)$ as $t \rightarrow+\infty$ and we claim that $\alpha=-\infty$. Indeed, otherwise we may define

$$
V:=\min _{t \in[0, T]}\left\{\sum_{k=1}^{M} \lambda_{k} r_{k}(t)\left(1-\frac{e^{\left(m_{k}-1\right) \alpha}}{1+e^{n_{k} \alpha}}\right)\right\}>0,
$$

and choose an arbitrary $\epsilon>0$ small enough such that $\sum_{k=1}^{M} \lambda_{k} r_{k}(t) \epsilon<\frac{V}{2}$ for all $t$. Fix $t_{1} \geq t_{0}$ such that $\frac{e^{m_{k} y\left(t-\tau_{k}(t)\right)-y(t)}}{1+e^{n_{k} y\left(t-\mu_{k}(t)\right)}}<\frac{e^{\left(m_{k}-1\right) \alpha}}{1+e^{n} k^{\alpha}}+\epsilon$ for all $t \geq t_{1}$, then from (13) we deduce:

$$
\begin{aligned}
y^{\prime}(t) & =\sum_{k=1}^{M} \lambda_{k} r_{k}(t) \frac{e^{m_{k} y\left(t-\tau_{k}(t)\right)-y(t)}}{1+e^{n_{k} y\left(t-\mu_{k}(t)\right)}}-b(t) \\
& \leq \sum_{k=1}^{M} \lambda_{k} r_{k}(t)\left(\frac{e^{\left(m_{k}-1\right) \alpha}}{1+e^{n_{k} \alpha}}+\epsilon\right)-b(t) \\
& <\sum_{k=1}^{M} \lambda_{k} r_{k}(t) e^{m_{k} B_{k}}\left(\frac{e^{\left(m_{k}-1\right) \alpha}}{1+e^{n_{k} \alpha}}-1\right)+\frac{V}{2} \\
& <-\frac{V}{2}
\end{aligned}
$$

a contradiction.

Case II. $y(t)$ is oscillatory. Suppose that $\lim \sup _{t \rightarrow+\infty} y(t)>-\infty$, then we may set $\left\{t_{j}\right\}_{j \in \mathbb{N}}$ such that $t_{j} \nearrow+\infty, y^{\prime}\left(t_{j}\right)=0, \lim _{j \rightarrow+\infty} y\left(t_{j}\right)=\lim _{\sup _{t \rightarrow+\infty}} y(t)=$ $\omega_{1} \in \mathbb{R}$. Define $\lim \sup _{j \rightarrow+\infty} y\left(t_{j}-\mu_{k}\left(t_{j}\right)\right):=\omega_{2} \leq \omega_{1}$.

If $\omega_{2}=-\infty$, then

$$
0=y^{\prime}\left(t_{j}\right) \leq \sum_{k=1}^{M} \lambda_{k} r_{k}\left(t_{j}\right) \frac{e^{m_{k}\left(B_{k}+y\left(t_{j}-\mu_{k}\left(t_{j}\right)\right)\right)-y\left(t_{j}\right)}}{1+e^{n_{k} y\left(t_{j}-\mu_{k}\left(t_{j}\right)\right)}}-b\left(t_{j}\right)
$$


thus,

$$
b\left(t_{j}\right) e^{y\left(t_{j}\right)} \leq \sum_{k=1}^{M} \lambda_{k} r_{k}\left(t_{j}\right) e^{m_{k} B_{k}} e^{y\left(t_{j}-\mu_{k}\left(t_{j}\right)\right)} .
$$

Next, take a large enough constant $S>0$. For $j \gg 0$, we obtain:

$$
b\left(t_{j}\right) e^{y\left(t_{j}\right)} \leq \sum_{k=1}^{M} \lambda_{k} r_{k}\left(t_{j}\right) e^{m_{k} B_{k}} e^{-S}
$$

and hence

$$
b_{m i n} e^{\omega_{1}-\epsilon} \leq \sum_{k=1}^{M} \lambda_{k} r_{k}\left(t_{j}\right) e^{m_{k} B_{k}} e^{-S} .
$$

This contradicts the fact that $\omega_{1} \in \mathbb{R}$. Now suppose $\omega_{2} \in \mathbb{R}$, then

$$
b\left(t_{j}\right) e^{y\left(t_{j}\right)} \leq \sum_{k=1}^{M} \lambda_{k} r_{k}\left(t_{j}\right) e^{m_{k} B_{k}} e^{y\left(t_{j}-\mu_{k}\left(t_{j}\right)\right)} .
$$

Let $\epsilon>0$, for $j$ large enough,

$$
b\left(t_{j}\right) e^{y\left(t_{j}\right)} \leq \sum_{k=1}^{M} \lambda_{k} r_{k}\left(t_{j}\right) e^{m_{k} B_{k}} e^{\omega_{2}+\epsilon}
$$

then

$$
\begin{gathered}
b\left(t_{j}\right) e^{\omega_{1}-\epsilon} \leq \sum_{k=1}^{M} \lambda_{k} r_{k}\left(t_{j}\right) e^{m_{k} B_{k}} e^{\omega_{2}+\epsilon} \\
e^{\omega_{1}-\epsilon} \leq e^{\omega_{2}+\epsilon}
\end{gathered}
$$

Since $\epsilon$ is arbitrary we deduce that $\omega_{1}=\omega_{2}$. Finally, define $V>0$ as in the previous case, with $\alpha=\omega_{1}=\omega_{2}$ and fix $\epsilon>0$ such that $\sum_{k=1}^{M} \lambda_{k} r_{k}\left(t_{j}\right) e^{m_{k} B_{k}} \epsilon<\frac{V}{2}$. As before, we deduce that $y^{\prime}\left(t_{j}\right)<-\frac{V}{2}$, a contradiction.

As a final remark, let us show that both in the case $m_{j}<1$ for some $j$ and the superlinear case, the autonomous problem admits positive equilibrium points. Indeed, assume that $b$ and $r_{k}$ are constant and $\sum_{k=1}^{M} \lambda_{k} r_{k} \leq b$. Let $f: \mathbb{R} \rightarrow \mathbb{R}$ given by $f(u)=\sum_{k=1}^{M} \lambda_{k} r_{k} \frac{e^{\left(m_{k}-1\right) y}}{1+e^{n} k^{y}}-b$; then it suffices to prove that $f$ has at least one zero. For the superlinear case (namely, $m_{j}>n_{j}+1$ for some $j$ ), it is seen that

$$
f(0)=\sum_{k=1}^{M} \lambda_{k} r_{k} \frac{1}{2}-b<\sum_{k=1}^{M} \lambda_{k} r_{k}-b \leq 0
$$


and

$$
\lim _{u \rightarrow+\infty} f(u)=+\infty
$$

so $f$ vanishes in $(0,+\infty)$. If $m_{i} \leq n_{i}+1$ for all $i$ and $m_{j}<1$ for some $j$, then we deduce as before that $f(0)<0$ and

$$
\lim _{u \rightarrow-\infty} f(u)=+\infty
$$

Thus, $f$ vanishes in $(-\infty, 0)$ and the conclusion follows.

\section{Conclusions}

By applying a theorem based on the continuation method, this paper provides sufficient conditions for existence and multiplicity of positive periodic solutions for a generalized hematopoiesis model. The results are new and complement previously known results.

It is observed that, in some particular cases, our methods guarantee the existence but not multiplicity of solutions. However, this fact does not automatically imply uniqueness; thus, it is an interesting open problem to analyze, for such cases, whether or not uniqueness of positive periodic solutions can be proved.

Acknowledgement. We thank the anonymous referees for insightful comments that led to an improvement of this manuscript.

\section{References}

[1] P. Amster, L. Idels, Periodic Solutions in general scalar non-autonomous models with delays, Nonlinear Diff. Equ. Appl. NoDEA 20, 1577-1596 (2013).

[2] L. Berezansky, E. Braverman, On existence of positive solutions for linear difference equations with several delays, Adv. Dyn. Syst. Appl. 1 No. 1, 29-47 (2006).

[3] D. BAI, Y. XU, Periodic solutions of first order functional differential equations with periodic deviations, Comput. Math. Appl. 53, 1361-1366 (2007).

[4] Y. Chen, L. HuAng, Existence and global attractivity of a positive periodic solution of a delayed periodic respiration model, Comput. Math. Appl. 49, 677687 (2005). 
[5] L. Glass, A. Beuter, D.Larocque, Time delays, oscillations, and chaos in physiological control systems, Math. Biosciences 90, 111-125 (1988).

[6] K. Gopalsamy, S.L. Trofimchuk, N.R. Bantsur, A note on global attractivity in models of hematopoiesis, Ukrainian Math. J. 50 No. 1, 3-12 (1998).

[7] F. HAn, Q. WANG, Existence of multiple positive periodic solutions for differential equation with state-dependent delays, J. Math. Anal. Appl. 324, 908-920 (2006).

[8] Y. Li, Y. Kunng, Periodic solutions of periodic delay Lotka-Volterra equations and systems, J. Math. Anal. Appl. 255, 260-280 (2001).

[9] G. Liu, J. YAn, F. Zhang, Existence and global attractivity of unique positive periodic solution for a model of hematopoiesis, J. Math. Anal. Appl. 334, 157171 (2007).

[10] J. D. Murray, Mathematical biology. I. An introduction, 3rd ed, SpringerVerlag, Berlin (2002).

[11] M.C. Mackey, L. Glass, Oscillation and chaos in physiological control systems, Science 197, 287-289 (1977).

[12] A.J. Nicholson, The balance of animal population, J. Animal Ecol. 2, 132178 (1933).

[13] S. PAdhi, S. SRIVASTAVA, Multiple periodic solutions for nonlinear first order functional differential equations with applications to population dynamics, Appl. Math. Comput. 203 1, 1-6 (2008).

[14] S. Padhi, S. SRIVAstava, J. Dix, Existence of three nonnegative periodic solutions for functional differential equations and applications to hematopoiesis, Panamer. Math. J. 19, 27-36 (2009).

[15] S. Padhi, S. Srivastava, S. PAti, Three periodic solutions for a nonlinear first order functional differential equation, Appl. Math. Comput. 216, 24502456 (2010).

[16] S.H. Saker, S. Agarwal, Oscillation and global attractivity in a nonlinear delay periodic model of population dynamics, Appl. Anal. 81, 787-799 (2002).

[17] A. Wan, D. JiAng, Existence of positive periodic solutions for functional differential equations, Kyushu J. Math. 56, 193-202 (2002). 
[18] A. Wan, D. JIAng, X. XU, A new existence theory for positive periodic solutions to functional differential equations, Comput. Math. Appl. 47, 12571262 (2004).

[19] W. Wang, B. LaI, Periodic solutions for a class of functional differential system, Archivum Math. 48 No. 2, 139-148 (2012).

[20] X. Wu, J. Li, H. ZHou, A necessary and sufficient condition for the existence of positive periodic solutions of a model of hematopoiesis, Comput. Math. Appl. $54,840-849$ (2007).

[21] D. Ye, M. Fan, H. WAng, Periodic solutions for scalar functional differential equations, Nonlinear Anal. 52, 1157-1181 (2005).

[22] W. Zhang, D. ZHu, P. Bi Existence of periodic solutions of a scalar functional differential equation via a fixed point theorem, Math. Comput. Model. 46, 718-729 (2007). 